\title{
Vers des systèmes optiques athermiques dans I'infrarouge
}

\author{
Katie SCHWERTZ, Ingénieur Conception, Edmund Optics \\ kschwertz@edmundoptics.com \\ Traduction et adaptation : Françoise MÉTIVIER
}

Pour les nombreuses applications sensibles aux variations de température, il est souvent important de pouvoir disposer de systèmes optiques dits athermiques, dont le comportement est indépendant : les modifications de température n'entraînent alors en particulier aucune variation de la focalisation. La conception de tels systèmes est particulièrement critique dans l'infrarouge, mais de nouvelles méthodes graphiques permettent d'aboutir à des doublets achrothermiques, qui sont à la fois athermiques et achromatiques.

\section{L'infrarouge, \\ un domaine critique}

Plusieurs facteurs rendent critique la conception de systèmes athermiques dans l'infrarouge. Le coefficient de dilatation thermique (CTE) des verres utilisés pour les applications dans l'infrarouge est plus important que celui des verres utilisés dans le domaine visible. Parallèlement, la variation de leur indice de réfraction en fonction de la variation de température est beaucoup plus importante que celle observée pour des verres du visible : une modification de la température de l'environnement induit donc une forte variation de l'indice de réfraction du verre. Enfin, le matériau du boîtier est également sensible à la variation thermique, et ses modifications mécaniques observées lors des changements de température doivent aussi être prises en compte dans la conception d'un système athermique.

\section{Déterminer \\ la défocalisation thermique}

La dilatation et la contraction d'un matériau en raison de changements de température sont régies par le coefficient de dilatation thermique du matériau, noté $\alpha$, qui est de l'ordre de grandeur de $10^{-6} \mathrm{~m} /{ }^{\circ} \mathrm{C}$ (ou ppm $/{ }^{\circ} \mathrm{C}$ ). La variation de la longueur $\mathrm{L}$ du matériau en raison d'une variation de la température est donnée par l'équation 1 (voir encadré pour toutes les équations utilisées dans cet article).

La défocalisation thermique d'une lentille dans l'air est définie comme la variation de la position du point focal sur l'axe lorsque la température varie. Cette variation de position est due, d'une part, à la variation de l'indice de réfraction et, d'autre part, à la dilatation du verre qui constitue la lentille (figure 1). Cette défocalisation est donnée par l'équation 2 , dans laquelle $\beta$ représente le coefficient thermo-optique de la lentille. $\beta$ peut être défini en utilisant l'équation 3 , dans laquelle $\alpha_{g}$ est le CTE du verre et $n$ son indice. En toute rigueur, l'équation donnant $\beta$ doit inclure un terme lié au changement de l'indice de réfraction de l'air, mais, dans l'infrarouge, ce terme est faible par rapportà la variation de la lentille elle-même et peut donc être négligé.

Pour une lentille montée dans un boîtier présentant un coefficient de dilatation thermique $\alpha_{h^{\prime}}$ la variation de la position du point focal est une combinaison de la variation de la distance focale de la lentille dans l'air et de la variation de la position du plan image due à l'expansion ou à la contraction du boîtier. La variation totale est alors donnée par l'équation 4.

On voit bien que, si la dé-focalisation due à la variation de la longueur du boîtier est égale et opposée à la variation de la mise au point de la lentille dans l'air, la dé-focalisation totale est alors nulle, et le système est considéré comme athermique.

\section{Doublets achromatiques et athermiques}

Parmi les systèmes optiques, un des plus utilisés est le doublet achromatique, qui utilise deux lentilles présentant des aberrations chromatiques égales et opposées. Le coefficient de dispersion

Équations utilisées dans cet article

Équation 1: $\Delta L=\alpha L \Delta T$
Équation 2: $\Delta f=\beta f \Delta T$
Équation 3: $\beta=\alpha_{\mathrm{g}}-\frac{1}{n-1} \frac{d n}{d T}$
Équation 4: $\Delta f=f\left(\beta_{\text {lens }}-\alpha_{\mathrm{h}}\right) \Delta T$
Équation 5: $v=\frac{n_{\text {mid }}-1}{n_{\text {short }}-n_{\text {long }}}$

Équation 6: $\frac{\Phi_{1}}{\Phi}=\frac{v_{1}}{v_{1}-v_{2}}$

Équation 7: $\frac{\Phi_{2}}{\Phi}=-\frac{v_{2}}{v_{1}-v_{2}}$

Équation 8: $\quad \frac{\Phi_{1}}{\Phi}=\frac{v_{\mathrm{T} 1}}{v_{\mathrm{T} 1}-v_{\mathrm{T} 2}}$

Équation 9: $\frac{\Phi 2}{\Phi}=-\frac{v_{\mathrm{T} 2}}{v_{\mathrm{T} 1}-v_{\mathrm{T}}}$

Équation 10: $\frac{v_{1}}{v_{\mathrm{T} 1}}=\frac{v_{2}}{v_{\mathrm{T} 2}}$

Pour ces équations, la puissance des différents composants du système est donnée au centre de la bande de longueurs d'onde utilisée et l'indice utilisé est celvi de la longueur d'onde de référence. 


\section{Defocus vs temperature}

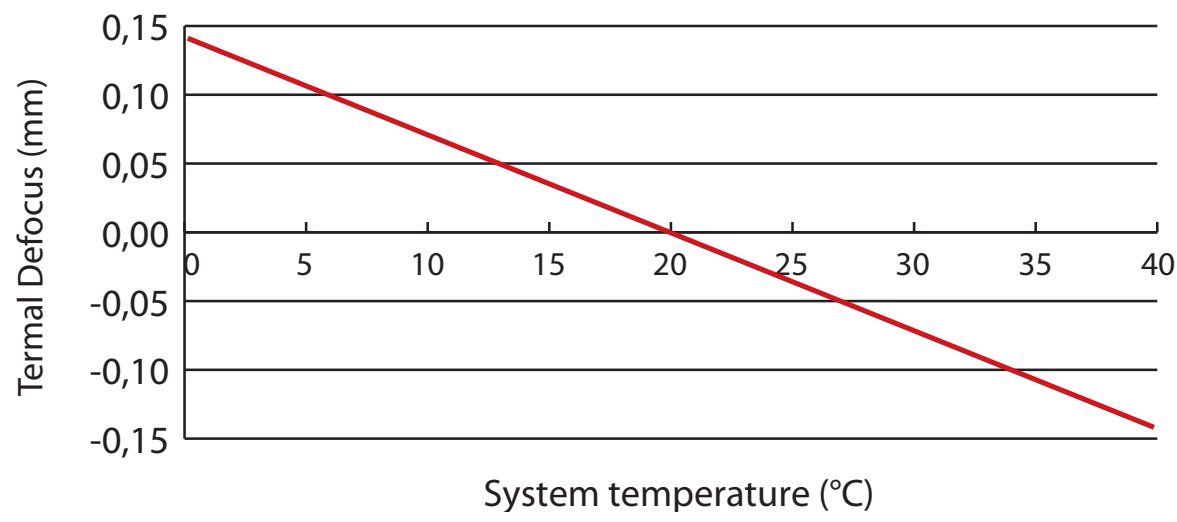

Figure 1. Défocalisation thermique d'une lentille simple en germanium dans l'air.

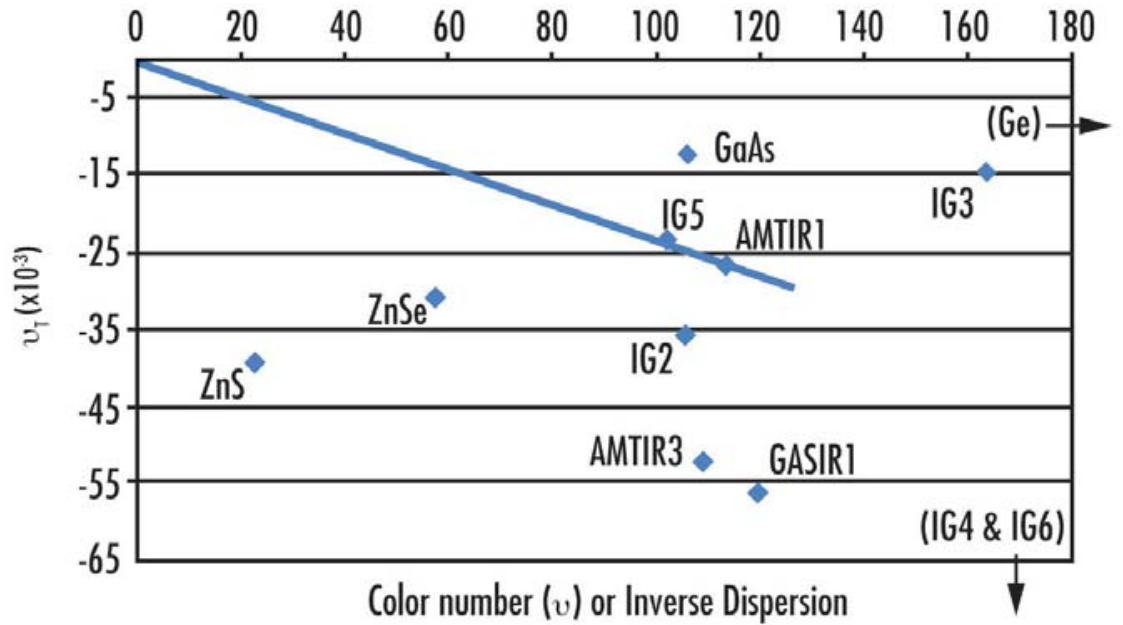

Figure 2. Méthode graphique pour déterminer un couple de verres susceptibles de permettre de réaliser un doublet achrothermique.

d'un matériau, noté $v$, est défini sur une bande de longueurs d'onde en fonction de l'indice de réfraction du matériau aux longueurs d'onde limites et à la longueur d'onde moyenne (équation 5). Un doublet est achromatique s'il satisfait à la fois les équations 6 et 7, dans lesquelles $\phi_{1}$ est la puissance de la lentille $1, \phi_{2}$ la puissance de la lentille 2 et $\phi$ la puissance du doublet.

De manière analogue, on peut utiliser l'inverse du coefficient thermo-optique, appelé généralement le $v$ thermique, dans les équations achromatiques pour concevoir un doublet athermique (équations 8 et 9). Si le doublet conçu satisfaitl'ensemble des équations de 6 à 9 , alors il est à la fois achromatique et athermique: on dit alors qu'il est achrothermique (équation 10).

En traçant le $v$ thermique $\left(v_{T}\right)$ par rapport au $v$ chromatique, on peut identifier visuellement deux matériaux qui peuvent être utilisés pour développer un système achrothermique. Compte tenu de l'équation d'une droite $(y=m x+b$, où $m$ est la pente et $b$ l'intersection avec l'axe des $y$ ), si l'intersection avec l'axe des y est fixée égale à zéro et que le matériau choisi présente un couple de coefficients $\left(v, v_{T}\right)$, la pente est $m=v_{T} / v$. D'après l'équation 10 , il faut que la pente des deux matériaux utilisés soit égale pour obtenir un système achrothermique : deux matériaux reliés par une droite passant par l'origine répondront donc aux conditions.

Comme le montre la figure 2 , les verres IG5 et AMTIR1 peuvent fournir une solution quasi achrothermique dans la bande 8 à $12 \mu \mathrm{m}$ (lointain infrarouge). Cette figure ne tient pas compte des modifications de longueur d'un boîtier éventuel.

\section{Prise en compte du boîtier dans le cas d'un doublet}

Une alternative à la méthode précédente consiste à tracer le coefficient thermo-optique $\beta$ par rapport à l'inverse du coefficient de dispersion v. Cette méthode permet non seulement d'identifier deux matériaux optiques permettant de réaliser un système achrothermique, mais contribue également à identifier le CTE du matériau du boîtier nécessaire pour que le système dans son boîtier constitue une solution achrothermique. Comme le montre la figure 3, l'intersection avec l'axe des y fournit le CTE du matériau du boîtier, grâce à la droite qui part de cette intersection et traverse deux matériaux. Dans le cas où le CTE ainsi défini ne correspond à aucun matériau, il est alors nécessaire d'utiliser un boîtier constitué de deux matériaux, ou une solution de montage mécanique.

\section{Cas de systèmes plus complexes}

Lorsque les méthodes précédentes ne permettent pas de réaliser des systèmes achrothermiques de façon simple, il est alors nécessaire d'utiliser des composants optiques plus complexes, comme des éléments diffractifs, ou d'augmenter le nombre de lentilles.

Rappelons que les méthodes décrites ne s'appliquent que dans le domaine infrarouge, domaine dans lequel la défocalisation due aux variations de l'indice de l'air suite à des changements de température peut être négligée.

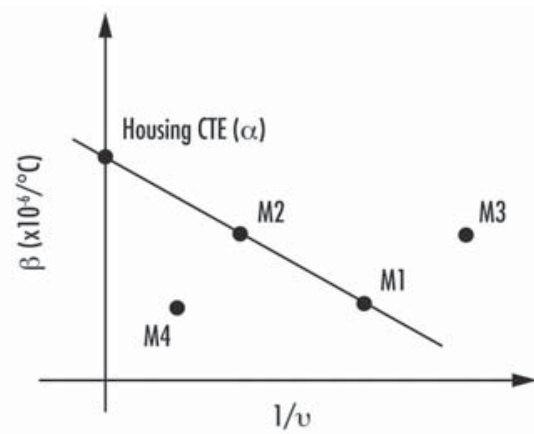

Figure 3. Méthode graphique permettant de déterminer le coefficient de dilatation thermique $d u$ matériau nécessaire pour réaliser, avec un doublet conçu avec les matériaux $M 1$ et $M 2$, une solution achrothermique. 